\title{
C3-BIOECONOMY
}

Circular and Sustainable Bioeconomy

\section{Factores habilitantes y limitantes para el desarrollo empresarial de la Bioeconomía Circular. Análisis empírico para el caso de Andalucía}

\author{
María M. Borrego-Marín¹, Julio Berbel², José A. Gómez-Limón³ y
}

Anastasio J. Villanueva ${ }^{4}$

Autor de Correspondencia: mbmarin@us.es

\section{Resumen:}

El presente trabajo tiene por objetivo analizar los factores habilitantes y limitantes para el desarrollo empresarial de iniciativas de éxito en el ámbito de la bioeconomía circular (BEC) en Andalucía. La metodología seguida para su realización ha consistido en la selección de una muestra de empresas andaluzas con líneas de negocio basadas en actividades de BEC, en la realización de entrevistas extensas a las personas gestoras para la identificación de los factores habilitantes y limitantes de tales líneas de negocio y en la agregación de la información cuantitativa recogida para la realización de un análisis descriptivo global y sectorial. Los principales resultados que se desprenden de dicho análisis es que los factores habilitantes que han fomentado el desarrollo de estas iniciativas han sido principalmente el 'Compromiso ético con el medio ambiente', seguido de cerca por el 'Conocimiento tecnológico específico' y la 'Rentabilidad privada'. Por su parte, aquellos factores que han actuado como limitantes han sido, en primer lugar, la 'Burocracia', seguida por la 'Normativa reguladora de la actividad' $y$, en igualdad de importancia, por las 'Necesidades financieras' y por las 'Barreras tecnológicas'.

Palabras clave: Bioeconomía, Economía circular, Andalucía, Barreras, Motivaciones, Empresas.

\section{Enabling and limiting factors for business development of the Circular}

\section{Bioeconomy. Empirical analysis for the case of Andalusia}

\author{
María M. Borrego-Marín ${ }^{1}$, Julio Berbel², José A. Gómez-Limón ${ }^{3}$ y
}

Anastasio J. Villanueva ${ }^{4}$

\section{Abstract:}

The present work aims to analyze the enabling and limiting factors for the successful business development in the field of circular bioeconomy (CBE) in Andalusia. The analysis relies on a survey of a selection of successful Andalusian companies with business lines based on CBE activities, based on extensive interviews to firm managers to identify the enabling and limiting factors of such business lines, and in the aggregation of the quantitative information collected to derive a basic global and sectoral descriptive assessment. The main results that emerge from this analysis is that,

\footnotetext{
1 Universidad de Sevilla y Grupo WEARE (España), mbmarin@us.es; https://orcid.org/0000-0003-2397-6041.

2 Universidad de Córdoba y Grupo WEARE (España), berbel@uco.es; https://orcid.org/0000-0001-6483-4483.

3 Universidad de Córdoba y Grupo WEARE (España), jglimon@uco.es; https://orcid.org/0000-0002-6364-0027.

4 IFAPA (Instituto para la Investigación y Formación Pesquera y Agraria) y Grupo WEARE (España), anastasioj.villanueva@juntadeandalucia.es; https://orcid.org/0000-0002-1384-8372.
}

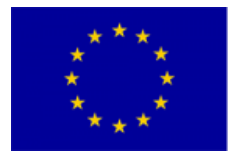

UNIÓN EUROPEA

PROYECTO COFINANCIADO POR EL FONDO EUROPEO DE DESARROL
(EEDER) Una manera de hacer Europa

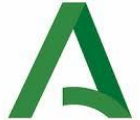

Junta de Andalucía
Andalucía

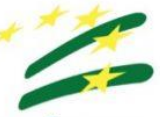

se mueve con Europa 
on the one hand, the enabling factors that have fostered the development of these initiatives have been mainly the 'Ethical commitment to the environment', closely followed by 'Specific technological knowledge' and 'Private profitability'. On the other hand, the factors that have acted as limiting factors have been, in the first place, the 'Bureaucracy', followed by the 'Regulations related to the activity' and, in equal level of importance, the 'Financial needs' and the 'Technological barriers'.

Key Words: Bioeconomy, Circular economy, Andalusia, Barriers, Limitations, Motivations, Business

\section{INTRODUCCIÓN}

La bioeconomía circular (BEC) integra los conceptos de bioeconomía y economía circular con la vocación de representar un modelo económico sostenible económica, social y ambientalmente (Carus y Dammer, 2018; Kardung et al., 2021). La bioeconomía engloba al conjunto de las actividades económicas relacionadas con la producción, transformación y utilización, directa $\mathrm{o}$ indirecta, de recursos de origen biológico con el fin de producir y transformar biomasa para el suministro de alimentos, piensos, materiales, energía y servicios demandados por los ciudadanos (EC, 2018). La economía circular es un modelo de producción, distribución y consumo en el que el valor de los productos, materiales y demás recursos permanece el mayor tiempo posible ( $p$. ej., reciclando, reparando, etc.), potenciando su uso sostenible y eficiente y reduciendo al mínimo la generación de residuos (EC, 2015; Ghosh, 2020). La BEC envuelve, por tanto, elementos comunes a ambos conceptos, como son la mejora del uso de los recursos y la ecoeficiencia, la reducción de la huella de carbono, la reducción de la demanda de carbono fósil y la valorización de los residuos (Carus y Dammer, 2018).

La BEC implica a múltiples sectores económicos ya existentes, tales como: agricultura, ganadería, silvicultura, pesca y acuicultura (dentro del sector primario); industria alimentaria, textil, papelera, química, farmacéutica y cosmética, biotecnológica y energética, entre otras (dentro del sector industrial); así como el sector de servicios asociados (consultoría, logística, etc.). Por lo tanto, al hablar de sector de la BEC, se consideran aquellas actividades económicas con base biológica que a su vez aplican los principios de la circularidad en sus procesos productivos. 
La Figura 1 ilustra el ámbito de estudio de la BEC.

Figura 1. Ámbito de la Bioeconomía Circular (BEC).

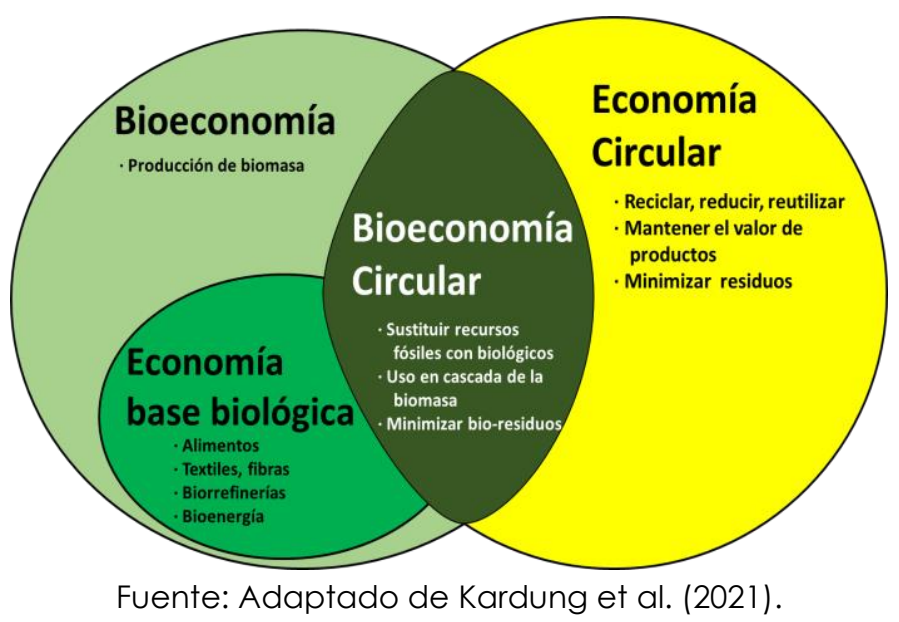

La expansión de la BEC es deseable para el conjunto de la sociedad, no solo por la generación de riqueza y empleo asociada a este sector, sino también por los beneficios ambientales que genera en forma de externalidades positivas (lucha frente al cambio climático, conservación de recursos naturales, etc.) y minimización de las externalidades negativas (p. ej., asociadas a la generación de residuos). La BEC está apareciendo en la agenda política cada vez con más intensidad, tanto a nivel internacional (OECD, 2018) y comunitario (EC, 2018), como a nivel nacional (MITECO, 2020) y autonómico (CAPDR, 2018). Las recientes iniciativas como el Pacto Verde Europeo (EC, 2019) y el nuevo plan de economía circular (EC, 2020) confirman el papel que se espera de la BEC en la Unión Europea del futuro, así como en cada una de sus regiones.

Con el fin de garantizar el adecuado uso de los recursos públicos, el apoyo de las diferentes administraciones implicadas en estas iniciativas políticas debe sustentarse en un correcto análisis del sector de la BEC. En este sentido, resulta muy útil examinar las actividades empresariales ya existentes, para conocer qué factores han facilitado y dificultado su desarrollo. Este análisis de factores habilitantes y limitantes resulta clave con el fin de apoyar la toma de decisiones públicas orientadas a diseñar e implementar instrumentos de política que fomenten de forma eficiente el crecimiento de este sector. En el caso de 
Andalucía, un estudio de este tipo resulta particularmente relevante habida cuenta del moderado desarrollo de este sector a nivel regional, sobre todo en el caso de actividades económicas de alto valor añadido, y del amplio potencial que este presenta considerando la gran producción de biomasa en la región y la importancia del tejido productivo asociado al sector primario y a la industria alimentaria. Este potencial ha quedado plasmado a nivel estratégico por parte de los decisores públicos a través de la Estrategia de Especialización Inteligente RIS3 de Andalucía, donde las Prioridades de Especialización Inteligente P3 "Aprovechamiento sostenible de los recursos endógenos de base territorial" y P6 "Investigación e innovación en agroindustria y alimentación saludable" están estrechamente relacionadas con el desarrollo de la BEC. En particular, las líneas de acción L32 "Nuevos procesos y productos para el aprovechamiento de los recursos agropecuarios" y L63 "Aprovechar las nuevas oportunidades en economía azul y economía verde", concretan el desarrollo de dichas prioridades estratégicas.

El presente análisis, por tanto, tiene por objetivo analizar los factores habilitantes y limitantes para el desarrollo empresarial de las iniciativas de BEC, utilizando Andalucía como caso de estudio.

\section{MÉTODO}

El enfoque metodológico seguido ha consistido en la realización de entrevistas extensas a una muestra de empresas andaluzas con líneas de negocio basadas en actividades de BEC, utilizando un cuestionario semiestructurado para la caracterización de dichas actividades, así como la identificación de los factores habilitantes y limitantes de tales líneas de negocio. La Figura 2 muestra la metodología de trabajo seguida, así como la programación de las distintas tareas realizadas a lo largo del año 2021. 
Figura 2. Metodología de trabajo seguida para la elaboración del estudio.

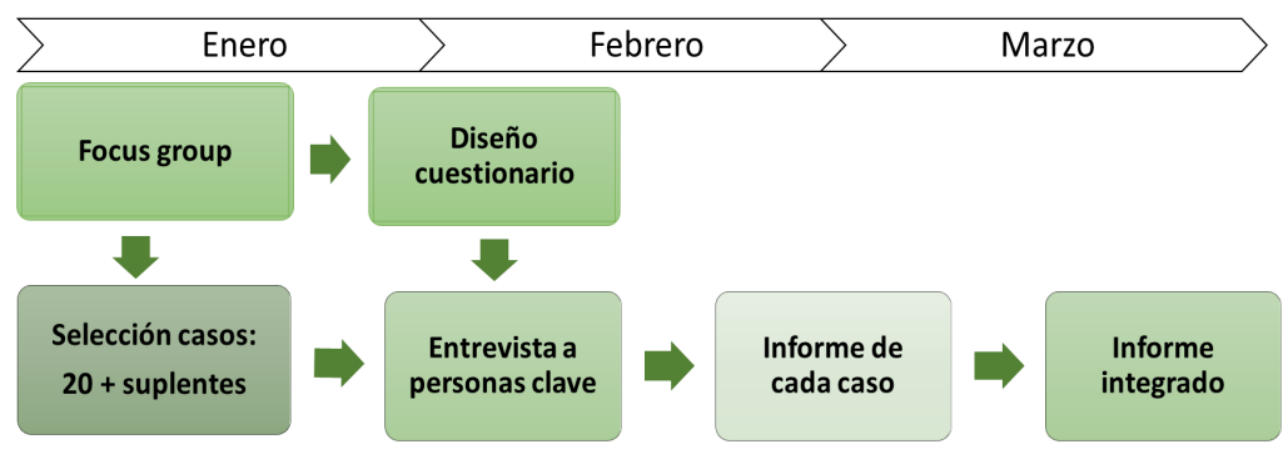

Fuente: Elaboración propia.

Para la selección de los casos de estudio, se establecieron los siguientes cuatro criterios:

1. Los casos de estudio a seleccionar deben ser empresas, como entes con personalidad jurídica responsables de la gestión de las actividades productivas.

2. Empresas que desarrollen líneas de negocio basadas en actividades de BEC, entendidas como la combinación de los conceptos de bioeconomía (actividades económicas relacionadas con la producción, transformación y utilización, directa o indirecta, de recursos de origen biológico) y economía circular (modelos de producción, distribución y consumo en el que el valor de los productos, materiales y demás recursos permanece el mayor tiempo posible), tal y como se muestra en la Figura 1.

3. Empresas que tengan localizadas sus operaciones de BEC en Andalucía, aunque su sede legal se sitúe fuera de esta Comunidad Autónoma.

4. Empresas comercialmente activas.

Sobre la base de estos criterios se buscaron las posibles empresas a analizar como casos de estudio a partir de las siguientes fuentes:

- Empresas que habían mostrado su interés en participar en el proceso de constitución del futuro Clúster de Bioeconomía de Andalucía (http://www.bioeconomiaandalucia.es/cluster-de-bioeconomia). 
- El catálogo de casos de éxito en prácticas de BEC elaborado como documento de apoyo para la redacción de la Estrategia Andaluza de Bioeconomía Circular (EABC).

- Búsqueda ad hoc realizada por el equipo investigador a partir de su experiencia previa en esta temática y de informaciones en internet y en prensa especializada.

- Aportaciones de las personas asistentes al focus group a partir de su experiencia y conocimiento previo. El focus group estuvo compuesto, además de por el equipo investigador, por personal de la Consejería de Agricultura, Ganadería, Pesca y Desarrollo Sostenible (CAGPDS) de la Junta de Andalucía (3), el Instituto para la Investigación y Formación Pesquera y Agraria (IFAPA) (2), la empresa Tecnología y Servicios Agrarios S.A (Tragsatec) (1), el Campus de Excelencia Internacional Agroalimentario (ceiA3) (1), la Universidad de Almería (1), la Fundación Cajamar (1), y de la consultoría independiente (2), todas ellas personas con amplia experiencia en temas de BEC.

Siguiendo este procedimiento, se seleccionaron un total de 35 iniciativas empresariales en Andalucía. Las personas integrantes del equipo de investigación intentaron contactar con las 35 empresas seleccionadas, y durante el proceso se descartaron 14 por diferentes motivos (ya no desarrollan líneas de negocio basadas en BEC, no se pudo contactar con ellas o, simplemente, porque no accedieron a ser entrevistadas). Finalmente, se entrevistaron un total de 21 iniciativas empresariales en Andalucía, repartidas entre las ocho provincias, que se clasificaron en base a su actividad económica, en los siguientes sectores de BEC presentes en la región: a) gestión, aprovechamiento y valorización de subproductos de la industria oleícola; b) gestión, aprovechamiento y valorización de subproductos del olivar; c) gestión, aprovechamiento y valorización de subproductos hortofrutícolas; d) gestión, tratamiento y reutilización de los recursos hídricos; e) actividades de BEC basadas en el uso de microalgas, insectos y fertilizantes; y f) aprovechamientos de alto valor añadido enmarcados dentro de la BEC. Las entrevistas tuvieron una duración media de 1,5 horas, realizándose entre febrero y marzo de 2021. 
La información cuantitativa recogida por los cuestionarios, en relación con la importancia relativa tanto de factores habilitantes como limitantes del desarrollo de las líneas de negocio de BEC, fue introducida en una base de datos para un análisis descriptivo básico. Dadas las limitaciones derivadas del escaso tamaño muestral $(n=21)$, no se ha podido realizar ningún análisis estadístico adicional para contrastación de hipótesis. En este sentido, la información cuantitativa anterior ha sido analizada por las personas autoras junto con la información cualitativa igualmente recogida en las entrevistas, al objeto de identificar patrones de respuestas comunes por sectores de actividad y derivar así las principales conclusiones del estudio.

\section{RESULTADOS}

\subsection{Factores habilitantes}

La literatura académica especializada (Sheppard et al., 2011; Wesseler y Braun, 2017) ha identificado diferentes fuerzas motrices o drivers detrás del desarrollo de las actividades de BEC. Kardung et al. (2021) clasifican estas fuerzas motrices en las siguientes categorías:

1. Tecnología e innovación: avances de ciencias biológicas, en tecnologías de la información y la comunicación (TIC) y otros avances tecnológicos sectoriales (p. ej., ingeniería de materiales).

2. Organización del mercado, que incluye el proceso de globalización y los procesos de integración empresarial, tanto horizontales como verticales.

3. Presión sobre los ecosistemas y el cambio climático (generación de residuos y fuentes de contaminación), que condiciona la disponibilidad de recursos naturales (p. ej., biomasa).

4. Preferencias en el consumo, que cada vez más valora los atributos relacionados con el medio ambiente incorporados en los bienes de consumo.

5. Desarrollo económico y demografía. 
Además, estas publicaciones destacan asimismo el papel clave de las políticas públicas en el desarrollo de las actividades de BEC, mediante múltiples tipos de incentivos y regulaciones recogidas en distintas políticas sectoriales (agraria, medio ambiente, I+D, etc.), con las que las entidades públicas tratan de dar respuesta a las actuales demandas sociales.

El cuestionario utilizado para las entrevistas ha tratado de recoger el papel de las fuerzas motrices, las demandas sociales y las políticas públicas como posibles factores habilitantes de las actividades de BEC en las empresas andaluzas. Para ello, se ha preguntado expresamente a las personas entrevistadas sobre una serie de motivaciones empresariales que explican el desarrollo de estas actividades, tal y como se reflejan en la Tabla 1.

Tabla 1. Factores habilitantes para la realización de negocios basados en BEC.

\begin{tabular}{llll}
\hline $\begin{array}{l}\text { Fuerza motriz / Demanda social / } \\
\text { Políticas públicas }\end{array}$ & Motivación empresarial \\
\hline Tecnología e innovación & $\bullet$ & Conocimiento tecnológico específico. \\
\hline Organización del mercado & $\bullet$ & $\begin{array}{l}\text { Rentabilidad privada } \\
\text { 'Reciclaje' de activos fijos disponibles }\end{array}$ \\
\hline Presión sobre los ecosistemas y & $\bullet$ & Compromiso ético con el medio ambiente \\
cambio climático & $\bullet$ & Necesidad en la gestión de residuos. \\
\hline Preferencias en el consumo & $\bullet$ & Imagen/reputación corporativa \\
\hline Políticas públicas & $\bullet$ & Apoyo de las administraciones públicas \\
\hline
\end{tabular}
Fuente: Elaboración propia.

En la Tabla 2, se puede apreciar la valoración media de la importancia de los diferentes factores habilitantes de actividades de BEC para el conjunto de empresas entrevistadas. 
Tabla 2. Importancia de los factores habilitantes para la realización de negocios basados en BEC.

\begin{tabular}{lcc}
\hline Factores habilitantes & $\begin{array}{c}\text { Importancia } \\
\text { media (0-10) }\end{array}$ & Ranking \\
\hline Compromiso ético con el medio ambiente & 8,7 & $1^{\circ}$ \\
Conocimiento tecnológico específico & 8,6 & $2^{\circ}$ \\
Rentabilidad privada & 8,3 & $3^{\circ}$ \\
Imagen/reputación corporativa & 7,6 & $4^{\circ}$ \\
Apoyo de las administraciones públicas & 5,1 & $5^{\circ}$ \\
Necesidad en la gestión de residuos & 5,0 & $6^{\circ}$ \\
Regulación ambiental obligatoria & 3,5 & $7^{\circ}$ \\
'Reciclaje' de activos fijos disponibles & 2,9 & $8^{\circ}$ \\
Otros motivos & 1,6 & $9^{\circ}$ \\
\hline
\end{tabular}

Fuente: Elaboración propia.

Como puede apreciarse, los tres factores habilitantes situados en las primeras posiciones de la tabla han recibido puntuaciones muy próximas, por lo que su importancia a la hora de desarrollar iniciativas de negocio basadas en BEC puede considerarse prácticamente similar. Aún así, la principal motivación declarada por las empresas entrevistadas para el desarrollo de actividades de BEC es el 'Compromiso ético con el medio ambiente', con una puntuación promedio de 8,7. Este resultado evidencia que la concienciación empresarial en favor del medio ambiente resulta un factor habilitante clave para la puesta en marcha de estas líneas de negocio. En segunda posición, dentro de las motivaciones para el desarrollo de actividades de BEC, se sitúa el 'Conocimiento tecnológico específico', con una puntuación muy similar a la anterior $(8,6)$. Este resultado no es sorprendente, ya que la implementación de estas actividades se basa de forma mayoritaria en nuevos conocimientos tecnológicos, principalmente de carácter biológico y bioquímico. La tercera motivación que puede considerarse igualmente como un factor habilitante clave es la 'Rentabilidad privada', que ha obtenido una puntuación promedio de 8,3. Este resultado evidencia la exigencia de una mínima rentabilidad de las actividades de BEC para su desarrollo, al igual que en cualquier otra actividad 
empresarial. Efectivamente, solo en el caso de que estas líneas de negocio resulten atractivas por su rentabilidad privada, estas van a poder atraer inversiones privadas que las desarrollen. Sin embargo, de la información cualitativa obtenida de las entrevistas, se deduce que muchas de las empresas analizadas no obtienen actualmente la rentabilidad privada suficiente de las actividades de BEC (menor rentabilidad que otras actividades de economía lineal). Y no solo por la actual coyuntura de crisis sanitaria y económica, sino por unos gastos de producción elevados y una fuerte competencia de otros productos competidores procedentes de actividades de economía lineal. De ello se deduce que las empresas del sector pueden estar pensando en el desarrollo de líneas de negocio basadas en la BEC como líneas rentables en un horizonte más a largo plazo, siendo conscientes que los niveles de rentabilidad privada deseados no se alcanzarán hasta pasado un periodo más o menos dilatado para su establecimiento y desarrollo.

En cuarto lugar, pero ya a cierta distancia de los anteriores, figura la motivación de la 'Imagen/reputación corporativa' (puntuación promedio de $7,6)$. En cualquier caso, y a diferencia de lo que ocurre con las tres motivaciones señaladas anteriormente, en relación con esta motivación deben señalarse importantes diferencias entre sectores de actividad. Así, en los casos de estudio relacionados con los subproductos de la industria oleícola, del olivar y hortofrutícola, la importancia relativa de esta motivación es similar a las anteriores, situándose entre los tres factores habilitantes más relevantes. Esta elevada importancia relativa tiene su explicación en el hecho de que las empresas de estos sectores producen bienes de consumo. En estos casos, una gestión ambientalmente responsable de los subproductos y residuos, más allá de las exigencias regulatorias, supone un atributo comercial que puede favorecer la distribución de sus productos principales (exigencias ambientales de las grandes cadenas de distribución alimentaria y concienciación ambiental del consumidor/a final). Por una razón similar, esta misma motivación figura como un importante elemento habilitante de los negocios de BEC en el sector de aprovechamientos basados en la producción de microalgas, insectos y fertilizantes, puesto que el desarrollo de sus productos en base a actividades de 
BEC es también un atributo comercialmente importante dentro de sus estrategias de marketing.

\subsection{Factores limitantes}

Por su parte, Gottinger et al. (2020) han realizado un estudio reciente, sobre el proceso de transición hacia iniciativas empresariales de BEC, basado en la literatura académica disponible, en el que han clasificado las principales barreras encontradas en seis categorías:

1. Política y regulación, que incluye barreras relacionadas con políticas o regulaciones existentes e inexistentes y problemas de implementación de políticas.

2. Tecnología y materiales, que engloba aspectos técnicos relacionados con la aplicación de tecnologías y el desarrollo de productos, así como a la disponibilidad de insumos materiales, estructuras de proveedores e infraestructuras físicas.

3. Condiciones de mercado e inversión, que involucran barreras relacionadas con la demanda y creación de mercado, y con la movilización y disponibilidad de recursos financieros.

4. Aceptación social, que contiene barreras relacionadas con la conciencia pública.

5. Conocimiento y redes, que incluye barreras relacionadas con la creación y aplicación del conocimiento y con la existencia y desarrollo de redes eficientes.

6. Rutinas y estructuras sectoriales, que contiene barreras relacionadas con la disposición y/o restricción política, los bloqueos que se desarrollan con el tiempo y desafíos relacionados con los estándares dominantes.

Además, su análisis muestra que la literatura existente identifica las barreras relacionadas con 'Conocimiento y redes', 'Rutinas y estructuras sectoriales', 'Políticas y regulaciones' y 'Mercado y condiciones de inversión' como las más relevantes, poniendo menos énfasis en las barreras relacionadas con 'Aceptación social' y 'Tecnología y materiales'. 
El cuestionario desarrollado para las entrevistas ha tratado de recoger las barreras o factores limitantes de las actividades de BEC en las empresas andaluzas. Para ello, se ha preguntado expresamente a las personas entrevistadas sobre una serie de aspectos que puedan afectar negativamente al desarrollo de estas. La Tabla 3 recoge dichos factores y muestra su vinculación directa con cinco de las categorías de barreras identificadas por Gottinger et al. (2020).

Tabla 3. Factores limitantes para el desarrollo de negocios basados en BEC.

\begin{tabular}{ll}
\hline Barreras & Factores limitantes de actividades de BEC \\
\hline Política y regulación & - Burocracia \\
\hline Tecnología y materiales & $\bullet$ Tecnológicos \\
\hline Condiciones de mercado e & - Financieros \\
inversión & Mercado-competencia \\
\hline Aceptación social & - Mercado-producto \\
\hline Conocimiento y redes & $\bullet$ Recursos Humanos \\
\hline
\end{tabular}

Fuente: Elaboración propia.

En la Tabla 4, por su parte, se puede apreciar la valoración media de la importancia de los diferentes factores limitantes de actividades de BEC para el conjunto de empresas entrevistadas.

Tabla 4. Importancia de los factores limitantes para el desarrollo de negocios basados en BEC.

\begin{tabular}{lcc}
\hline Factores limitantes & $\begin{array}{c}\text { Importancia } \\
\text { media (0-10) }\end{array}$ & Ranking \\
\hline Burocracia & 7,5 & $1^{\circ}$ \\
Normativa reguladora de la actividad & 6,1 & $2^{\circ}$ \\
Financieros & 5,8 & $3^{\circ}$ \\
Tecnológicos & 5,8 & $3^{\circ}$ \\
Recursos humanos & 4,0 & $5^{\circ}$ \\
Logística & 3,8 & $6^{\circ}$ \\
Mercado-competencia & 3,8 & $6^{\circ}$ \\
Mercado-productos & 3,1 & $8^{\circ}$ \\
Otros motivos & 1,3 & $9^{\circ}$ \\
\hline
\end{tabular}

Fuente: Elaboración propia. 
Como puede apreciarse, la principal barrera es la 'Burocracia', con una puntuación promedio de 7,5, seguida con más de un punto de diferencia por la 'Normativa reguladora de la actividad', con una puntuación promedio de 6,1, y, con una puntuación muy similar, por las barreras vinculadas a la 'Tecnología' y a la 'Financiación', que comparten el tercer puesto, con una puntuación promedio de 5,8. Esta jerarquía evidencia que las ideas de negocio vinculadas a la BEC sufren las consecuencias de un sistema administrativo que obstaculiza su desarrollo, dada la lentitud en los plazos y la resolución de los trámites y permisos, la complejidad de los mismos, así como la fragmentación competencial dentro de las propias administraciones. Asimismo, las personas entrevistadas han coincidido en que el desarrollo normativo va en muchos casos por detrás en el tiempo de las iniciativas innovadoras, que las actividades de BEC requieren necesariamente procesos de $1+D+i$ para superar carencias de conocimientos tecnológicos vinculadas a la innovación de procesos y productos, y que, en la mayoría de las ocasiones, dichos procesos requieren inversiones muy elevadas en $1+D+i$.

Un análisis sectorial de la importancia relativa de estos factores limitantes entre los sectores de actividad de las iniciativas andaluzas consultadas refleja un elevado grado de homogeneidad. Así, puede apreciarse que, en todos los sectores analizados, son los cuatro factores mencionados anteriormente, 'Burocracia', 'Normativa reguladora de la actividad', 'Tecnología' y 'Financiación', los que adquieren una mayor importancia como aspectos limitantes en el desarrollo de las iniciativas empresariales, a excepción del sector hortofrutícola, donde el factor 'Mercado-competencia' adquiere una importancia relativa similar a las barreras vinculadas a las necesidades de 'Financiación', y el sector de aprovechamientos de alto valor añadido, donde los 'Recursos humanos' aparecen como tercer factor limitante más importante, después de la 'Burocracia' y la 'Normativa reguladora de la actividad'. 


\section{CONCLUSIONES}

La BEC andaluza se encuentra en una fase inicial de su desarrollo en la mayoría de los sectores de actividad, si bien se observa cómo está progresando gradualmente. Se especializa sobre todo en aprovechar la biomasa local existente, como demuestra la alta presencia de empresas vinculadas a la biomasa generada por el olivar y la hortofruticultura, lo que justifica a su vez que estos subproductos se gestionen localmente, normalmente mediante procesos de BEC tradicionales (compostaje y valorización energética). La mayoría de las iniciativas son posibles por la elevada dimensión de la empresa y su posición competitiva dentro de su actividad principal. La idea en todos los casos de este sector es conseguir el 'residuo cero' y la generación de valor a partir de la biomasa residual.

Aún así, también existen iniciativas empresariales andaluzas emergentes con productos de mayor valor añadido, incluyendo compuestos utilizados en cosmética (p. ej., escualeno), en la construcción (building-blocks), bioestimulantes, microalgas, alimentación funcional (tanto animal como humana), entre otros productos. Se trata de empresas con un crecimiento importante, denominadas 'gacelas'. Además, también son destacables las iniciativas vinculadas a la reutilización del agua y la conversión de las EDAR (estaciones depuradoras de aguas residuales) en 'biofactorías', muestra de la competitividad del sector del agua en Andalucía, derivado de la escasez del recurso y su alta competitividad.

No obstante, también cabe destacar que a nivel regional están ausentes empresas del tipo 'biorrefinerías'. En la Unión Europea se estima en 800 el número de biorrefinerías con distintos niveles de madurez (comercial, demostración, piloto, I+D), pero la mayoría se localizan en la proximidad de los grandes puertos de aprovisionamiento de materias primas (commodities). Es por tanto natural que Andalucía, por su naturaleza periférica y lejanía a estos puertos (p. ej., Rotterdam, Hamburgo), se haya centrado en aprovechar su propia biomasa. No obstante, el modelo de 'biorrefinería' y la obtención de mayores compuestos 
de distinto grado de valor a través de enfoques productivos en cascada, es una asignatura pendiente de la BEC andaluza.

Se puede concluir por tanto que este "nuevo" sector se encuentra en una fase incipiente de desarrollo en Andalucía, en la medida en que está fuertemente sustentado en el conocimiento y la innovación. Además, su expansión es deseable para el conjunto de la sociedad, no solo por la generación de riqueza y empleo asociada a este sector, sino también por los beneficios ambientales que genera. Por ello, el apoyo público para promover el desarrollo del sector de la BEC está plenamente justificado, en la medida en que tal desarrollo derivará en una mejora del bienestar del conjunto de la ciudadanía.

De ahí la importancia del presente estudio, que indaga sobre los factores habilitantes y limitantes para el éxito de iniciativas empresariales enmarcadas dentro de la bioeconomía circular para el caso de estudio de Andalucía. Del análisis realizado se derivan varias recomendaciones que podrían ser tenidas en cuenta por las administraciones públicas para promocionar este nuevo sector, entre las que cabe destacar la simplificación de trámites, la armonización legislativa a nivel nacional, impulsar acciones colaborativas (p. ej., clústers y hubs de empresas), facilitar la inversión (p. ej., fomentando modelos de capitalriesgo), o el apoyo específico a PYMEs (p. ej., apoyando evaluación y certificación ambiental).

Por último, la principal limitación que se ha identificado en el estudio ha sido el escaso tamaño muestral utilizado, que no ha permitido realizar ningún análisis estadístico adicional. Por tanto, las investigaciones futuras deben ir encaminadas fundamentalmente a la ampliación de la muestra, con el fin de validar o no los resultados de este primer análisis y extraer conclusiones adicionales a partir de un análisis estadístico de mayor profundidad. 


\section{AGRADECIMIENTOS}

Este informe ha sido elaborado por el equipo de investigación del grupo WEARE (http://www.uco.es/investiga/grupos/weare/), con sede en la Universidad de Córdoba, por encargo de la Secretaría General de Agricultura, Ganadería y Alimentación de la Consejería de Agricultura, Ganadería, Pesca y Desarrollo Sostenible de la Junta de Andalucía, como parte del proyecto europeo POWER4BIO (https://power4bio.eu/, GA 818351).

\section{REFERENCIAS}

CAPDR (Consejería de Agricultura, P.y.D.R. (2018). Estrategia Andaluza de Bioeconomía Circular. Consejería de Agricultura, Pesca y Desarrollo Rural, Junta de Andalucía, Sevilla. Recuperado de: https://www.juntadeandalucia.es/export/drupaljda/Estrategia_Andaluz a Bioeconomia_Circular_EABC 18.09.2018.pdf

CARUS, M. y DAMMER, L. (2018). The "circular bioeconomy" - Concepts, opportunities and limitations. nova-Institut, Hürth (DE). Recuperado de: file:///Users/usuario/Downloads/External Resource Nova Paper 9 The C ircular Bioeconomy.pdf

EC (European Commission). (2015). Closing the loop - An EU action plan for the circular economy. $\operatorname{COM}(2015) 614$ final. European Commission, Brussels. Recuperado de: https://eur-lex.europa.eu/legalcontent/EN/TXT/?uri=CELEX:52015DC0614

EC (European Commission). (2018). A sustainable Bioeconomy for Europe: Strengthening the connection between economy, society and the environment. COM(2018) 673 final. European Commission, Brussels. Recuperado de: https://eur-lex.europa.eu/legalcontent/en/ALL/? uri=CELEX\%3A52018DC0673

EC (European Commission). (2019). The European Green Deal. COM(2019) 640 final. European Commission, Brussels. Recuperado de: https://ec.europa.eu/info/sites/default/files/european-green-dealcommunication en.pdf 
EC (European Commission). (2020). A new Circular Economy Action Plan For a cleaner and more competitive Europe COM/2020/98 final. European Commission, Brussels. Recuperado de: https://eur-lex.europa.eu/legalcontent/EN/TXT/? aid=1583933814386\&uri=COM:2020:98:FIN

GHOSH, S.K. (Ed.) (2020). Circular Economy: Global Perspective. Springer Nature, Singapore.

GOTTINGER, A., LADU, L. \& QUITZOW, R. (2020). "Studying the transition towards a circular bioeconomy-A systematic literature review on transition studies and existing barriers". Sustainability, 12(21), 8990. http://dx.doi.org/10.3390/su12218990

KARDUNG, M., CINGIZ, K., COSTENOBLE, O., DelAHAYE, R., heIJMAN, W., LOVRIĆ, M., VAN LEEUWEN, M., M'BAREK, R., VAN MEIJL, H., PIOTROWSKI, S., RONZON, T., SAUER, J., VERHOOG, D., VERKERK, P.J., VRACHIOLI, M., WESSELER, J.H.H. \& ZHU, B.X. (2021). "Development of the circular bioeconomy: drivers and indicators". Sustainability, 13(1), 413. http://dx.doi.org/10.3390/sul3010413

MITECO (Ministerio para la Transición Ecológica y Reto Demográfico). (2020). España Circular 2030. Estrategia Española de Economía Circular. MITECO, Madrid. Recuperado de: https://www.miteco.gob.es/es/calidad-y-evaluacionambiental/temas/economia-circular/espanacircular2030 defl_tcm30509532.PDF

OECD (Organization for Economic Cooperation and Development). (2018). Meeting Policy Challenges for a Sustainable Bioeconomy. OECD Publishing, Paris. Recuperado de: https://www.oecd.org/publications/policy-challenges-facing-asustainable-bioeconomy-9789264292345-en.htm

SHEPPARD, A.W., GILLESPIE, I., HIRSCH, M. \& BEGLEY, C. (2011). "Biosecurity and sustainability within the growing global bioeconomy". Current 
C3-BIOECONOMY, Revista de Investigación y Transferencia en Bioeconomía Circular y Sostenible N² (2021)

C3-BIOECONOMY

Opinion in Environmental Sustainability, 3(1), 4-10. http://dx.doi.org/https://doi.org/10.1016/j.cosust.2010.12.011

WESSELER, J. \& BRAUN, J.V. (2017). "Measuring the bioeconomy: economics and policies". Annual Review of Resource Economics, 9(1), 275-298. http://dx.doi.org/10.1146/annurev-resource-100516-053701 